\title{
Interculturalidad y pedagogía diferenciada: senderos compartidos*
}

\author{
Guillermo Rojas Trujillo \\ Universidad Distrital Francisco José de Caldas, Bogotá, Colombia. \\ Email: grojas@udistrital.edu.co
}

Resumen: A partir del reconocimiento de problemas curriculares y pedagógicos que presentan instituciones educativas observadas en la ciudad de Bogotá, se da a conocer la justificación y la trascendencia de la educación intercultural y su correspondiente pedagogía diferenciada para abordar problemas de calidad, integración, participación, pertinencia y democratización de dichas escuelas.

Palabras claves: Educación, Escuela, interculturalidad, pedagogía diferenciada, democracia escolar.

\section{Interculturality and differentiated pedagogy: shared footpaths}

\begin{abstract}
From the recognition of curricular and pedagogical problems presented by educative institutions observed in the city of Bogota, this essay discloses the justification and the importance of intercultural education and its corresponding differentiated pedagogy for the approach to problems of quality, integration, participation, relevance and democratization of these schools.
\end{abstract}

Key words: Education, school, interculturality, differentiated pedagogy, school democracy.

\section{Pedagogia intercultural e diferenciada: caminhos compartilhados}

Resumo: Após do reconhecimento de problemas curriculares e pedagógicas que as instituições educacionais têm visto na cidade de Bogotá, revela-se a justificativa ea importância da educação intercultural ea correspondente pedagogia diferenciada para tratar de questões de qualidade, integração, participação, relevância e democratização das escolas.

Palavras-chave: Educação, escola, multiculturalismo, pedagogia diferenciada, democracia escolar.

\section{Realidad educativa regional}

La educación en Colombia no dista mucho de lo ocurrido en los otros países del continente. Modelos escolares verticales que han estado acompañados por políticas de aumento de cobertura, de homogenización de procesos curriculares y de gestión, por la feminización de la matrícula, por las acciones orientadas a retener a los estudiantes de menores recursos en el sistema y, a tratar de que avancen a ciclos más avanzados. Esto no ha sido casual, están al margen de los procesos de calidad, equidad y relevancia educativa. Según Torres (2008), estas acciones se corresponden con la agenda neoliberal, que trae tras de sí, la privatización de la educación pública, la reducción del gasto público y la estandarización de contenidos, procesos de gestión, modelos de evaluación y resultados esperados. Al decir de este autor, "Unidad en la diversidad de experiencias educativas en la región puede ser la divisa de esta conversación sobre grandezas y miserias en la educación latinoamericana en el siglo XX” (Torres, 2008: 215).

Pero las políticas educativas también se relacionan con lo que ocurre al interior de las instituciones y las aulas de clase, con los procesos pedagógicos que son al final de cuentas los que configuran la formación de los sujetos y de las sociedades que tenemos. Ese es el propósito de esta reflexión.

A partir de observaciones de campo en algunas instituciones escolares de la ciudad ${ }^{1}$, son muchas las reflexiones que se derivan. Tanto en la Universidad Distrital Francisco José de Caldas ${ }^{2}$, como en el Colegio Policarpa Salavarrieta ${ }^{3}$, existen realidades multiculturales pero no educación intercultural, ya que hay expresiones culturales distintas, pero éstas no interactúan entre sí, sino que existen y tienen por separado sus propias dinámicas. Desde las instancias directivas no hay reconocimiento de esta diversidad, y menos 
acciones curriculares y pedagógicas para transformar sus espacios educativos. Son instituciones educativas que tienen currículos, al decir de Mora (2008), como plan de estudios y como planificación y organización educativa convencional. Su planeación, organización y práctica académica es vertical y segmentada, y está orientada a jóvenes y, niños, niñas y adolescentes con una visión occidental hegemónica. Supuestamente, las mismas estarían ausentes de los conflictos sociales, políticos, culturales y cognitivos que es común hoy en la ciudad de Bogotá y del mundo.

Lo anterior es lo común en el sistema de educación pública del país, muchas de las excepciones se presentan generalmente en el sector privado de la educación, como se puede observar en el ideario y práctica del Colegio Nómade ${ }^{4}$. En la medida que la educación que prestan las instituciones públicas, sobre todo las de nivel primario y secundario, las cuales son las que tienen mayor cobertura en el país, en ellas se debe seguir investigando sus procesos, pero simultáneamente se deben formular acciones curriculares y pedagógicas en el sentido de los requerimientos de niños y niñas del presente y del futuro.

\section{Multiculturalidad e interculturalidad en la escuela}

Hay que trabajar por una educación que responda a las necesidades de los contextos nacionales e internacionales, caracterizados por la diversidad y el multiculturalismo, la globalización y la planetarización, la comunicación internacional y por la presencia de los dispositivos digitales tecnológicos, entre las principales. Al decir de Gadotti (2003), los niños y niñas del presente y del futuro necesitan de una educación para la diversidad, con una ética de la diversidad y con una cultura de la diversidad. "Una sociedad multicultural debe educar un ser humano multicultural, capaz de oír, de prestar atención a lo diferente, respetarlo" (p. 49). En ese sentido, el proyecto pedagógico y el currículo de la institución escolar debe contemplar, además de la diversidad de niños y niñas y docentes, la dimensión de lo interdisciplinario, porque las realidades que viven los sujetos de la educación son integrales y complejas. A partir de ver y estudiar los fenómenos como integralidad y unidad se podría llegar a la transdisciplinariedad, lo que presupone la articulación del saber, el conocimiento, la vivencia, la emoción, el deseo, la escuela, la comunidad, el medio ambiente, el país y el mundo, en perspectiva a que el niño y niña trabajen solidaria y colectivamente. Además, el maestro y la escuela deben tener en cuenta lo internacional, lo universal, para que los niños comprendan la relación entre su localidad y el mundo, para que aspiren a vivir en una sociedad planetaria responsable.

Como agregado a estas dimensiones, en perspectiva de una educación nueva, Gadotti (2003) dice que el proyecto pedagógico político de la escuela debe tener autonomía para formular, ejecutar y evaluar sus planes; así mismo, la gestión democrática para que la escuela sea un laboratorio de cultura democrática que tanta falta hace a estas sociedades patriarcales. Esta democracia en la escuela debe estar orientada a cambiar la mentalidad de los directivos, de la comunidad educativa, de los padres de familia y de la sociedad local; porque la democracia debe formar para la ciudadanía activa y social y, para reflexionar y transformar sus procesos institucionales, pedagógicos y didácticos. En últimas, respecto a la escuela, Gadotti (2003) expresa que:

La diversidad cultural es la riqueza de la humanidad. Para cumplir su tarea humanista, la escuela necesita mostrar a los alumnos que existen otras culturas además de la suya. La autonomía de la escuela no significa aislamiento o cerrazón en una cultura particular. Escuela autónoma significa escuela curiosa, osada, que busca dialogar con todas las culturas y concepciones del mundo... pluralismo significa, sobre todo, diálogo con todas las culturas a partir de una cultura que se abre a los demás (p. 51).

Lo anterior es coherente con lo que plantea Freire (2005) sobre la práctica educativa en condiciones de una escuela transformada, sobre el rol de los actores, los procesos y los objetivos de la misma. Esta práctica educativa debería estar contextualizada y permanentemente reflexionada por los docentes y demás actores del acto pedagógico:

Recapitulando entonces: no hay práctica educativa sin sujetos, sin sujeto educador y sin sujeto educando; no hay práctica educativa fuera de ese espacio-tiempo que es el espacio-tiempo pedagógico; 
no hay práctica educativa fuera de la experiencia de conocer que técnicamente llamamos experiencia gnoseológica, que es la experiencia del proceso de producción del conocimiento en sí; no hay práctica educativa que no sea política; no hay práctica educativa que no esté envuelta en sueños; no hay práctica educativa que no involucre valores, proyectos, utopías. No hay entonces, práctica educativa sin ética (p. 42,43).

Desde hace mucho tiempo se viene insistiendo sobre la necesidad que la escuela se transforme para que interprete las realidades del mundo presente y las particularidades de niños y niñas latinoamericanos en sus contextos, que son diversos y complejos. Pero no se trata de transformar la escuela sin tener claro hacía donde se va. No es el cambio por el cambio, en donde todo vale, como observa Freire (2005). Desde la modernidad se ha dicho que los cambios sociales y de la escuela deben estar regidos por principios y horizontes previamente configurados por los actores comprometidos. No es a partir de las modas pasajeras o las narrativas comerciales del momento como se debe reformar la educación. Como por ejemplo, en muchas ciudades latinoamericanas los gobernantes y directivos escolares del momento han venido expresando la necesidad de la educación bilingüe, pero entienden lo bilingüe sólo como el aprendizaje del idioma inglés, dejando de lado las lenguas y demás expresiones de distintas culturas de sus respectivas ciudades y países.

Colombia es un país diverso por su realidad social, política y cotidiana, y multicultural por la presencia de más de 80 grupos o comunidades culturales a lo largo de todo su territorio. Esto se expresa en forma muy nítida en las grandes ciudades, como Bogotá, y por ende se refleja en las instituciones educativas, en las cuales en la medida que el tema no es reconocido y abordado, es fuente de múltiples conflictos. La Constitución Política de 1991 reconoce que el país es pluriétnico y multicultural, que se debe respetar los derechos de los pueblos indígenas, y hace una manifestación expresa de las garantías que el Estado debe proveer en materia social, política, económica y jurídica a todos sus ciudadanos en condiciones de igualdad, pero no igualitariamente. A partir de este fundamento, se oficializa la jurisdicción especial indígena, las lenguas de los grupos étnicos y se institucionaliza la participación de las comunidades en la dirección y administración de su educación para garantizar el respeto y desarrollo de su identidad cultural, como se expresa en la Ley General de Educación de 1994.

El pluralismo cultural constituye la respuesta política al hecho de la diversidad cultural. De esa forma, la Declaración Mundial de la Diversidad Cultural aprobada por la UNESCO en 2001, establece el respeto y reconocimiento internacional a la diversidad étnica y cultural en los diferentes ámbitos de las sociedades y la relación de esta dimensión con los derechos humanos, la ciudadanía y la democracia. Pero la diversidad en las sociedades no está referida exclusivamente a lo étnico, sino que además de éste, la diversidad hace alusión a la multiplicidad cultural de los ciudadanos y las comunidades, muchas de las cuales no pueden expresarse porque los canales institucionales, ciudadanos y escolares no se lo permiten.

Además de lo étnico hay que tener en cuenta a las poblaciones en condición de desplazamiento, las comunidades de LGTB (lesbianas, gay, transexuales y bisexuales), las denominadas "tribus urbanas", entre otras. Esta realidad de la multiculturalidad debe ser reconocida y aceptada activamente por la Escuela, ya que la misma cuando no se aborda adecuadamente es fuente de tensiones y conflictos y, además puede ser un factor incidente en las dificultades en las realizaciones y gestión de las propias instituciones. Su desconocimiento por directivos o docentes arbitrarios y hegemonistas en las instituciones son una clara violación de los Derechos Humanos. Por eso la escuela no puede ser ajena a los procesos pedagógicos en perspectiva de diversidad, interculturalidad y género.

En perspectiva de tener clara la posición que se asume en este texto, Soriano (2004), quien dirige un grupo de investigación sobre el tema en la Universidad de Almería, dice que:

La interculturalidad implica la creación y asunción de actitudes entre todos los miembros de la comunidad educativa que favorezcan la convivencia entre personas de diferentes etnias, culturas y razas. Pero las actitudes no se aprenden estudiando las lecciones de un libro, sino que se crean y potencian actuando diariamente de manera adecuada y coherente con el modelo de educación que se pretende. Por eso la escuela se convierte en un espacio privilegiado para potenciar la tolerancia, la convivencia entre los pueblos, e inculcar el valor positivo de la diversidad, siendo la respuesta a la 
misma uno de sus retos fundamentales en el siglo XXI. La escuela es un lugar de encuentro y de construcción de las trayectorias del profesorado y del alumnado en un marco político y cultural (p. 10).

La escuela es como el escenario en donde se encuentran las culturas y su deber es permitir la interacción entre las mismas y formar a los alumnos para que esto sea reconocido y practicado posteriormente en sus roles sociales y políticos. Las escuelas en ese sentido deben tener un carácter intercultural, democrático, autónomo, flexible y ético.

Para Meléndez (2008), apoyándose en Hidalgo (2006), el auge de la exigencia intercultural en la escuela y en la sociedad se presenta con el advenimiento de la posmodernidad, como un intento de revertir el proceso uniformizante dado en la modernidad, y de construir una sociedad pluralista en donde todas las identidades culturales sean posibles. La educación intercultural se presenta como una posibilidad de revertir el proceso de desnaturalización que la sociedad y el mismo ser humano ha sufrido al cabo de miles de años, por la imposición de visiones únicas y excluyentes sobre la vida y el mundo. Se puede deducir que la diversidad es genética y la desigualdad es cultural. Lo natural se puede ver que es diverso en donde caben todas las posibilidades de vida, pero la cultura y sobre todo la occidental, impuso una concepción y práctica unidimensional que llevó a que la diversidad fuera perseguida y negada. Por ese camino han sido eliminados muchos seres, comunidades e invisibilizadas muchas culturas. La conciencia planetaria que se consolida en el presente por los evidentes problemas de la tierra y por las crisis de las sociedades debido a los altos índices de violencia y todo tipo de pobrezas debe llevar a pensar en la necesidad de que el ser humano valore e imite las condiciones de vida biopsicosociales.

Otra idea importante que surge de estas reflexiones es que, la propuesta de Interculturalidad es un proyecto social y político, que presupone valores, actitudes y acciones definidas y puede ser una vía para alcanzar esa anhelada democracia, sueño de muchas generaciones a lo largo de la historia de la humanidad. Si se concibe y acepta que la interculturalidad presupone una plena aceptación de la diversidad y en esa medida los individuos pueden participar en las acciones y decisiones que toman las sociedades y comunidades (se incluye la comunidad educativa) para construir un mundo que sea crisol de todas las culturas, tenderán a desaparecer los actos de imposición, de homogenización o de eliminación de los otros. La democracia no descansa tanto en que unos gobiernen y otros obedezcan, sino en que todos se puedan autogobernar. La propuesta de la interculturalidad es una ayuda a los teóricos y gobernantes que se han devanado la cabeza pensando sobre los modelos y formas para alcanzar la deseada democracia. La misma vida biológica y de otros seres muestra que ellos coexisten porque albergan dentro de sí la diversidad y la contradicción y no necesitan de otros para que los gobiernen.

En ese sentido sustenta sus ideas Olnech (2000), quien tomando los desarrollos Bourdieuianos, expresa que la educación intercultural y en sí las sociedades interculturales son sociedades que tienen mayor capital cultural. Lo intercultural sí contribuye a elevar el capital cultural de las sociedades, pero como lo mismo ha sido negado por muchos gobiernos y sociedades de países de la región, entonces esto podría ser una razón para explicar, a excepción de lo socioeconómico, otras pobrezas de las mismas sociedades. Cuando una sociedad reconoce lo intercultural, es una sociedad que toma conciencia histórica y social de lo que es y actúa en consecuencia. Por el contrario, en estos países periféricos prevalece una visión colonizada de lo social y lo cultural, pensando más en la cultura anglosajona como paradigma de realización.

\section{Emergencia de la Pedagogía Diferenciada}

La educación intercultural necesita de su correlato en la dimensión pedagógica. Ya que sería insuficiente que sólo se reformara la organización y la gestión escolar, desconociendo lo que ocurre al interior de las aulas y en los espacios educativos colectivos de la institución, es decir de la práctica educativa, a decir de Freire (2005). Esta práctica educativa debe estar basada en la pedagogía diferenciada.

Para Flórez (1997), la pedagogía es una disciplina humanista que reelabora y comparte campos con otras disciplinas del hombre. Es como una especie de supersaber, "que estudia y propone estrategias para lograr la transición del niño del estado natural al estado humano, hasta su mayoría de edad, como ser 
racional autoconsciente y libre” (p. XXXII)). Este ha sido una gran aspiración moderna de la pedagogía, formar seres humanos autónomos y con mayoría de edad, en el sentido de la expresión Kantiana.

A su vez, para Meirieu (2009) la pedagogía es la "reflexión acerca de la educación del niño y, por extensión, también, acerca de la educación del adulto, en la medida en que para este último la génesis no cesa con la juventud" (p. 224). Esto está en concordancia con la concepción clásica de la pedagogía como la disciplina de la educación. Pero en otro texto, el profesor Meirieu (2004) habla de la pedagogía diferenciada, la cual debe tener en cuenta las diferencias de los niños y que las agrupaciones de los mismos también deben tener en cuenta lo que tienen de común con los otros niños. Así mismo, que esta pedagogía no puede considerársele como muy nueva. Dice textualmente:

"Diferenciar la pedagogía" no es pues, en modo alguno, una "revolución" en la Escuela, ni, con mayor motivo, una renuncia a los principios, o incluso a la existencia de una institución que se diluiría, entonces, en una gran cantidad de procesos individuales yuxtapuestos. "Diferenciar la pedagogía" es, al contrario, reforzar la institución escolar reintegrando en su seno lo que nunca debería haber relegado a la esfera privada, familiar o comercial: el seguimiento individualizado de los alumnos. "Diferenciar la pedagogía" es dar a cada uno los medios para hacer suyos los saberes respetando sus necesidades específicas y acompañándolo en las mejores condiciones en su proceso de aprendizaje (p. 156).

Es decir, reconocer la diversidad cultural en la escuela, lo cual no es nada nuevo, presupone tener en cuenta las particularidades de los niños y niñas en diversas dimensiones, pero sin perder la perspectiva de universalidad que tiene la escuela por los conocimientos científicos y por la necesidad de la democracia. No es parcelar, fragmentar o balcanizar la institución o el aula de clase, sino que el maestro se relaciona con individualidades en tránsito a ser sujetos, a ser universales. Esto presupone aceptación, reconocimiento, solidaridades, participaciones diversas y el cuidado para todos en las interacciones humanas.

La pedagogía diferenciada es para reconocer la multiplicidad de realidades y expresiones culturales que traen consigo los educandos, los docentes y padres de familia dentro de la escuela, para no caer en la pretensión de que una cultura ahogue o le imponga sus valores a las otras. Se trata de no abandonar el sentido y los logros de la modernidad, la cual según Touraine (2005) tiene rasgos destacados en la razón ilustrada y los derechos universales de los sujetos. Esta modernidad es un horizonte de realización de la escuela, la cual no debería permitir perder.

“... si la escuela pública debe conceder una importancia cada vez mayor a la comunicación intercultural, al mismo tiempo debe reforzar los principios de la modernidad, no es para dejarse pisotear por asociaciones religiosas tan a menudo asociadas a partidos políticos, étnicos o religiosos, sino, al contrario, para facilitar el acceso de todos, y por tanto de todas las minorías, a la modernidad haciendo posible la combinación de ésta con experiencias individuales y colectivas que mezclan en sus recorridos culturas históricamente situadas y principios universales" (Touraine, 2005: 223).

Para Perrenoud (2007) la pedagogía diferenciada, como una opción militante, proviene de las falencias de la escuela actual, expresadas en el fracaso escolar y en las desigualdades a que están sometidos los alumnos. A partir de una visión hegemónica, en donde se aplica el mismo rasero a todos los niños, la escuela no establece las diferencias reales del capital cultural que tienen los alumnos, y cree que las jerarquías de excelencia, que son las representaciones sociales que se tienen sobre ciertas desigualdades, las consideran como reales y significativas, cuando son creadas precisamente por las construcciones sociales colectivas. Por eso dice el autor, que en la escuela hay una indiferencia ante las diferencias de individuos y comunidades.

En el sentido de que los desvalidos o expulsados de los escenarios educativos formales y hegemónicos precisan de otro tratamiento, Bárcena y Mélich (2000) expresan la necesidad de que la educación sea considerada como un acto ético, y en consecuencia proponen que la educación es como un acontecimiento ético, o la pedagogía de la radical novedad. Esta concepción pedagógica está orientada a construir unas relaciones en la práctica educativa que sea expresión de una ética de la vigilancia o de la atención, pero no para la retaliación o la venganza, sino para estar atentos al presente y se pueda soñar y garantizar un mejor porvenir. Como no es una pedagogía del poder, por eso no busca que los vencidos sean vencedores, sino que 
es, construir un mundo sin exclusiones. Para construir relaciones donde no sólo prevalezca la autonomía, sino que la heteronomía tenga sentido en la medida que los individuos dependen unos de otros y por eso debe estar atenta a la suerte de los otros. Es una educación como acompañamiento, del abrazo, hospitalidad y recibimiento del otro en su radical alteridad; pensar la educación como acción que se encarna en la natalidad y la creación de novedad, en inspiración de las ideas Arendtianas.

En esta pedagogía la heteronomía y la razón anamnética son dos principios medulares. Pero estos no pretenden negar,

"la importancia de la autonomía, la capacidad del diálogo y de la razón comunicativa para la resolución de nuestros conflictos. Su único propósito es completarlos e indicar, con toda la seriedad y el rigor que el asunto merece, que después de dos guerras que han asolado una Europa que hoy se persigue unida y defensora de los derechos humanos, y que después de una guerra como la que desoló los Balcanes, no es posible defender una idea de la libertad y del dialogo inspirada en la autoridad de una Razón que no pudo evitar la barbarie, y que deberíamos situar en la entraña misma de nuestras prácticas de formación, la responsabilidad incondicional hacía el otro y el recuerdo de las víctimas" (Bárcenas y Mélich, 2000: 32).

Esta concepción está en correspondencia con la pedagogía de los abrazos, la cual se ha venido planteando a partir de la experiencia docente y de la indagación sobre la realidad violenta colombiana, Rojas (2009). En referencia a esta pedagogía se dijo lo siguiente:

Entonces, la pedagogía de los abrazos es una acción educativa en sentido real y simbólica que busca que los niños y niñas sensibilicen y hagan conciencia sobre sus condiciones de vida que han estado marcados por la violencia, para que reconozcan a otras personas que no son fuente de violencia sino de apoyo, afecto y respeto, para que vean que la vida puede ser diferente al legado de violencia que traen tras de sí y que está incorporada a su cuerpo, a su pensamiento y a sus prácticas intersubjetivas. Es una pedagogía de la esperanza porque puede ser un camino para salir del laberinto de la violencia que ha acompañado a los colombianos por muchos siglos; es una pedagogía de la espera, porque debe permitir en el tiempo y en el espacio que los niños y las niñas renazcan con sus ritmos en un ambiente y en unas relaciones de respeto $\mathrm{y}$, es una pedagogía diferenciada, porque debe formar a los estudiantes y profesores en el reconocimiento que la diversidad es una característica de lo genético, biológico, psicológico, social, político, étnico, sexual y cultural que es propio de las sociedades humanas. Y es una pedagogía que reconoce la dimensión política de la educación en la medida que se puede pensar y soñar en unas relaciones interpersonales fraternas, en sujetos autónomos pero con sentido colectivo y, en una sociedad democrática que vaya más allá de las manidas mayorías obtenidas en eventos eleccionarios y se piense la misma más como encuentros de diversidades, más como estilo de vida y como proyecto que como fin en sí mismo. (p. 9).

Esta es una forma de pedagogía diferenciada, y cuya concepción intenta integrar los aportes hechos por muchos teóricos y maestros en ejercicio que desde las más disímiles latitudes vienen contribuyendo a la aspiración de lograr sociedades más democráticas, participativas, solidarias, incluyentes y éticas, para lo cual se precisa de una nueva educación en donde lo intercultural sea destacado y se tenga en cuenta lo que sugiere Mora (2008), respecto a que se necesitan nuevos modelos curriculares, y mayor participación de otros actores de la educación, como los estudiantes, los padres y la misma comunidad. Así mismo, que es necesario contar con otros saberes, como los populares, originarios, universales y la misma complejidad social, a la cual no puede escapar la escuela. Propone que se tengan en cuenta estos ocho conocimientos en las escuelas: personales, populares, socioculturales, originarios, universales, comunitarios, transformadores y escolares, como una forma de decir que la institución escolar no debe regirse solo por los conocimientos académicos que están enlazados a los conocimientos científicos, los cuales finalmente dieron origen a las áreas y materias en que se ha fragmentado el conocimiento en las instituciones escolares.

\section{Interculturalidad y pedagogía diferenciada para transformar la escuela}


Se puede observar la importancia que tiene la interculturalidad y la pedagogía diferenciada para construir nuevas escuelas que respondan a las necesidades de las sociedades contemporáneas y, de las comunidades que históricamente han estado marginadas o invisibilizadas por la visión hegemónica y vertical occidental que se ha instituido en las esferas gubernamentales y en los consejos directivos de las instituciones educativas. Es una visión que desde el etnocentrismo del mundo anglosajón ha invisibilizado a culturas étnicas ancestrales y pretende deificarse como la única posibilidad de desarrollo y de progreso. Ante esta agresión, las culturas minoritarias y autóctonas de la región han respondido también con intenciones de negar las elaboraciones y logros universales.

Al reconocer esta realidad, se podrían responder muchos interrogantes relativos a la calidad educativa, a la pertinencia de los currículos, a la democratización de los espacios escolares, a la integración social y a la formación ética y ciudadana de los niños y niñas de nuestros países, los cuales han estado regidos por políticas educativas inspiradas en los organismos económicos internacionales, quienes tienen solo una visión de mundo hegemónica y unilateral, distante sobremanera de las riqueza cultural de los individuos y comunidades de este continente.

\section{Notas}

\footnotetext{
* Este texto fue presentado inicialmente a un curso del Doctorado Latinoamericano de Educación de la Universidad de Costa Rica, el cual cursa el autor, en el año 2009. Esta versión se presenta con algunas modificaciones efectuadas para esta publicación.

${ }^{1}$ Estas reflexiones se escriben luego de efectuar algunas visitas a instituciones educativas de la ciudad, en el segundo semestre de 2009.

${ }^{2}$ Universidad Pública de la ciudad de Bogotá, en la cual trabaja el autor.

${ }^{3}$ Institución pública de educación primaria y secundaria de la ciudad

${ }^{4}$ Institución privada relativamente nueva de la ciudad, que como proyecto innovador ofrece educación primaria con énfasis en el arte y lo ambiental.
}

\section{Bibliografía}

Bárcena, Fernando y Mélich, Joan-Carles (2000), La educación como acontecimiento ético. Natalidad, narración y hospitalidad, Paidós, Barcelona.

Flórez, Rafael (1997), Hacía una pedagogía del conocimiento, McGraw-Hill, Bogotá.

Freire, Paulo (2003), El grito manso, Siglo XXI editores, Buenos Aires.

Gadotti, Moacir y colaboradores (2003), Perspectivas actuales de la educación, Siglo XXI editores, Buenos Aires.

Hidalgo, Liliam (2006), “Educación e interculturalidad: entre la diversidad y la desigualdad”, La Piragua, Revista Latinoamericana de educación y política $\mathrm{N}^{\circ} 24$.

Meirieu, Philippe (2009), Aprender, sí. Pero ¿cómo?, Octaedro, Barcelona.

Ídem (2004), En la escuela hoy, Octaedro, Barcelona.

Meléndez, Lady (2008), La transformación intercultural desde los ámbitos de aprendizaje, XIV Congreso Internacional de Tecnología y Educación a Distancia. De la práctica educativa hacía la inclusión sociocultural, Del 5 al 7 de noviembre del 2008. Universidad Estatal a Distancia, Costa Rica, San José.

Mora, David (2008), "Concepción crítica del concepto de currículo. Ideas para la transformación, diseño y desarrollo curricular en tiempos de cambios sociopolíticos”, Integra Educativa No.2.

Olnech, M. (2000), "Can multicultural Education change what counts as cultural capital". American Educational Research Journal, 37 (2), 317-348. 
Perrenaud, Philippe (2007), "De la indiferencia ante las diferencias a las pedagogías diferenciadas", en Pedagogía Diferenciada, editorial popular, España.

Rojas, Guillermo (2009), Pedagogía de los Abrazos: un camino para abordar la violencia en Colombia. Ponencia presentada al Congreso Internacional "Presente y futuro de la profesión docente en Colombia", 4, 5 y 6 de Octubre 2009, Bogotá.

Soriano, Encarnación (2004), La práctica educativa intercultural, La Muralla, Madrid.

Torres, Carlos Alberto (2008), "Después de la tormenta neoliberal: la política educativa latinoamericana entre la crítica y la utopía”, Revista Iberoamericana de Educación, N. 48 (2008), pp. 207-229.

Touraine, Alain (2005), Un nuevo paradigma para comprender el mundo de hoy, Paidós, Barcelona.

$$
* * *
$$

Recibido: 26.04.2011

Aceptado: 20.07.2011 